\title{
Stress-strain analysis of a toric pipe for inner pressure loads
}

\author{
David Ferràs ${ }^{\mathrm{a}, \mathrm{b}, *}$, Dídia I.C. Covas ${ }^{\mathrm{a}}$, Anton J. Schleiss ${ }^{\mathrm{b}}$ \\ anstituto Superior Técnico, University of Lisbon, Lisboa, Portugal \\ ${ }^{\mathrm{b}}$ Laboratory of Hydraulic Constructions (LCH), École Polytechnique Fédérale de Lausanne, Switzerland
}

\section{A R T I C L E I N F O}

\section{Article history:}

Received 11 March 2014

Accepted 28 July 2014

\section{Keywords:}

Fluid-structure interaction

Hydraulic transient

Membrane theory of shells of revolution

Stress-strain analysis

\begin{abstract}
A B S T R A C T
This research aims to enhance the knowledge on stress-strain states of a coiled pipe used for hydraulic transient experiments. The membrane theory of shells of revolution has been applied for the description of axial and circumferential strains while an inverse method has been used to analyse bending effects. The developed stress-strain model has been successfully validated for dynamic loading. Finally, a simplified version of the stress-strain equations is presented with the goal to facilitate fluid-structure interaction FSI implementation in the hydraulic transient model. The fluid wave speed formula for coiled pipes has been presented.
\end{abstract}

(c) 2014 Elsevier Ltd. All rights reserved.

\section{Introduction}

The aim of the present research is to achieve a better understanding of the stress-strain states of a coiled pipe during hydraulic transient events. Classic theory of water-hammer assumes that the pipe does not move along its axis and that the circumferential deformation is incorporated, together with pipe deformability and fluid (liquid) compressibility, in the elastic wave speed. However, several physical phenomena not taken into account in classic water-hammer theory affect transient pressure wave attenuation, shape and timing (Bergant et al., 2008a,b). One of these effects, Fluid-Structure Interaction analysis (FSI), can be important depending on the set up of the piping system. Consequently, a FSI analysis has to be carried out in order to determine the effect of the structural inertia over the transient pressure wave. Pipe systems experience severe dynamic forces during water-hammer events. When these forces make the system move, significant FSI may occur, so that liquid and pipe systems cannot be treated separately and interaction mechanisms must be taken into account (Tijsseling, 2007). Recommendations by means of some dimensionless parameters and with the aim to analyse when FSI must be taken into account are drawn in Tijsseling (1996).

Coiled pipes have many industrial engineering applications. Pipe coils are used in most heat exchange systems, like cooling systems in power plants, industrial and commercial refrigerators, solar water heaters or radiators for auto-motive industry. To the knowledge of the authors, the incorporation of the pipe coil behaviour in hydraulic transient analysis through FSI has never been carried out. The purpose of the paper is to find a relationship that describes the pipe coil deformation as a function of pressure changes, in order to incorporate it in a hydraulic transient solver.

* Corresponding author. 


\section{Background theory}

A stress-strain analysis is a first step for FSI, the goal is to determine the pipe deformation equations. Torsion, bending, shear and axial stresses and strains are the structural responses that a piping system may experience during water-hammer events. In classic water-hammer theory, FSI coupling is implicitly included by applying dimensionless coefficients to the ratio between fluid compressibility and pipe elasticity in the wave speed calculation in order to account for different pipe constraints. For instance, for a thin-walled straight conduit with expansion joints throughout its length and a linear-elastic behaviour of the pipe wall the referred coefficient is 1 (Chaudhry, 1987). Although radial expansion of the pipe-wall is taken into account, axial strains are not considered in classic water-hammer theory. Skalak (1955) extended classic water-hammer theory aiming to include axial stress and movement of the pipe. Circumferential strain in straight pipes is described by the following relation and included in the conservation equations:

$$
\epsilon_{c}=\frac{1 p r}{E} \frac{p}{e}
$$

where $\epsilon_{c}$ is the circumferential strain, $E$ is the Young's modulus of elasticity of the pipe, $p$ is the inner pipe pressure, $r$ is the pipe radius, and $e$ is the pipe-wall thickness.

However, in coil systems, the structural behaviour considerably differs from that of a straight pipe, either in axial and circumferential directions, due to the toroid geometry and the cross-section shape, which becomes oval when the pipe is curved. Consequently, when classic water-hammer theory is applied in coiled pipes discrepancies generally arise changing the wave shape and overshooting computed pressures during peak transitions. Anderson and Johnson (1990) analysed the effect of tube ovalling on pressure wave propagation speed in the context of physiological flows, reaching to the conclusion that transient pressure waves are very sensitive to the eccentricity of an ovalled cross-section.

A toroid can be described as a surface of revolution, i.e. by rotation a plane curve through 360 over a straight line (axis of revolution) in the plane of the curve. A coiled pipe can be geometrically defined in the same manner but adding a constant slope in the direction of the axis of revolution, and the number of coil rings will depend on the angle of rotation. Therefore, the helical system of a coil can be considered as a composition of toroids and described as a thin shell of revolution. Membrane theory of shells of revolution is a suitable approach to solve circumferential and axial strains in an axisymmetrically loaded torus. However, in a torus with ovalled or elliptic cross-section, when it is pressurized, loads are not axisymmetric and bending moments are generated. Membrane theory of shells of revolution assumes that no bending moments, twisting moments and transverse shearing forces that exist in the shell (Zingoni, 1997). Bending theory of shells must be applied to account for bending effects and to describe the complete state of stress and strains. However, such theory is more general and, consequently, its main equations are more difficult to be solved for complex geometries.

Clark and Reissner (1950) proposed a methodology based on the Boltzmann superposition principle to describe stressstrain states in Bourdon tubes. Such approach consisted essentially of the computation of axial and circumferential strains using the thin-walled assumption and describing the bending effects using the thick-walled assumption and applying bending theory. In the context of hydraulic transients, Brown and Tentarelli (2001a,b) carried out FSI coupling in order to account for the Bourdon effect during water-hammer events. The structural constraint conditions of the pipe coil, though, do not comply with the Bourdon tube theory, as Bourdon tube is a disconnected torus with closed ends, while the pipe coil analysed must be considered as a connected torus. However, a similar approach can be applied in order to determine its stress-strain states, combining thin and thick-walled assumptions by the Boltzmann superposition principle as a function of the applied loads. The current paper approaches the stress-strain states problem in pipe coils by computing circumferential and axial strains using membrane theory of shells of revolution and the bending effects by applying an inverse approach based on thick-walled assumption.

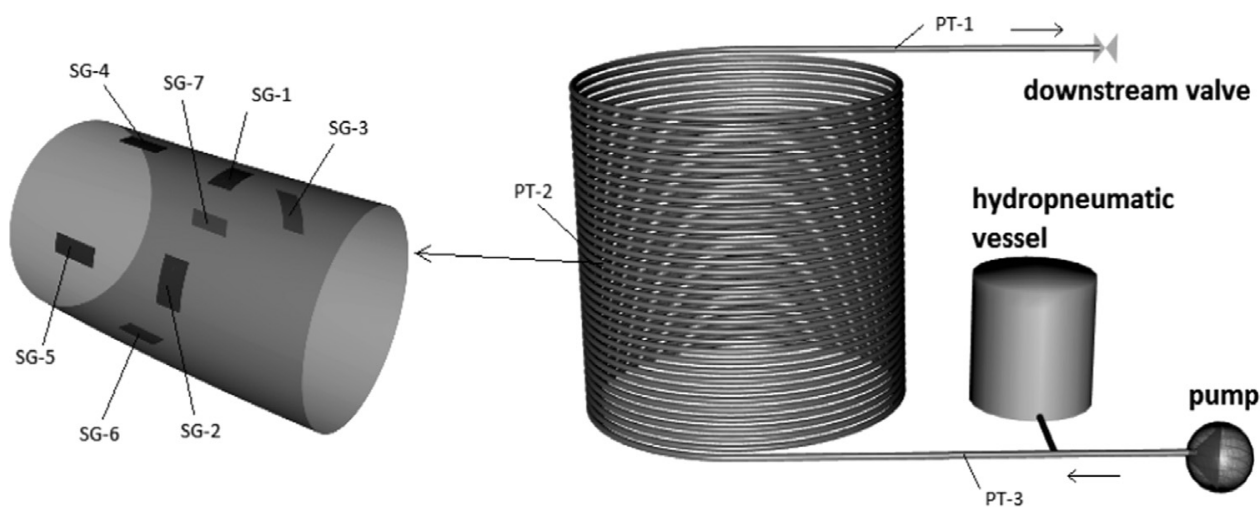

Fig. 1. Scheme of the copper pipe coil facility used for the experimental data collection (right) and detail of strain-gauges installation (left). 
(a)

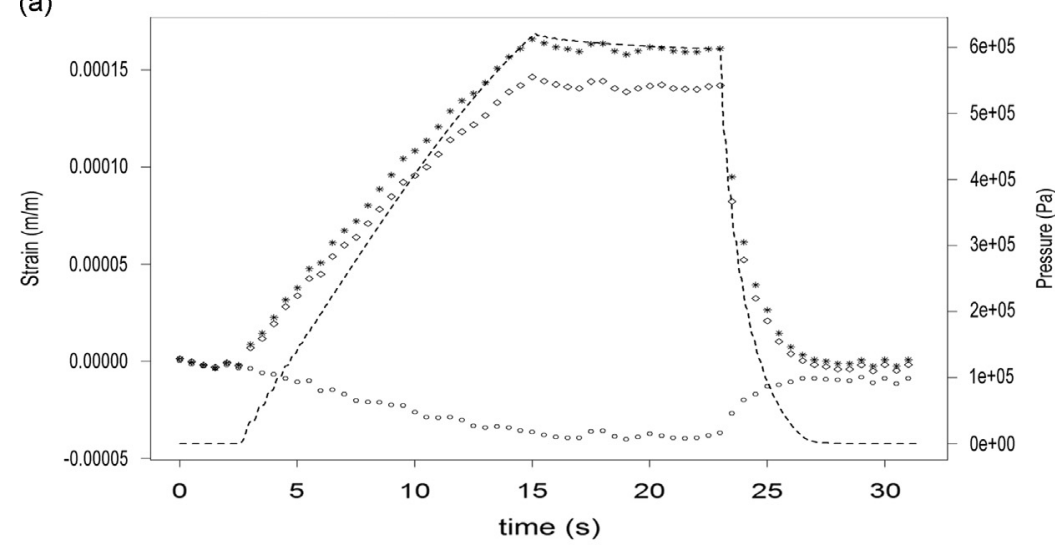

(b)

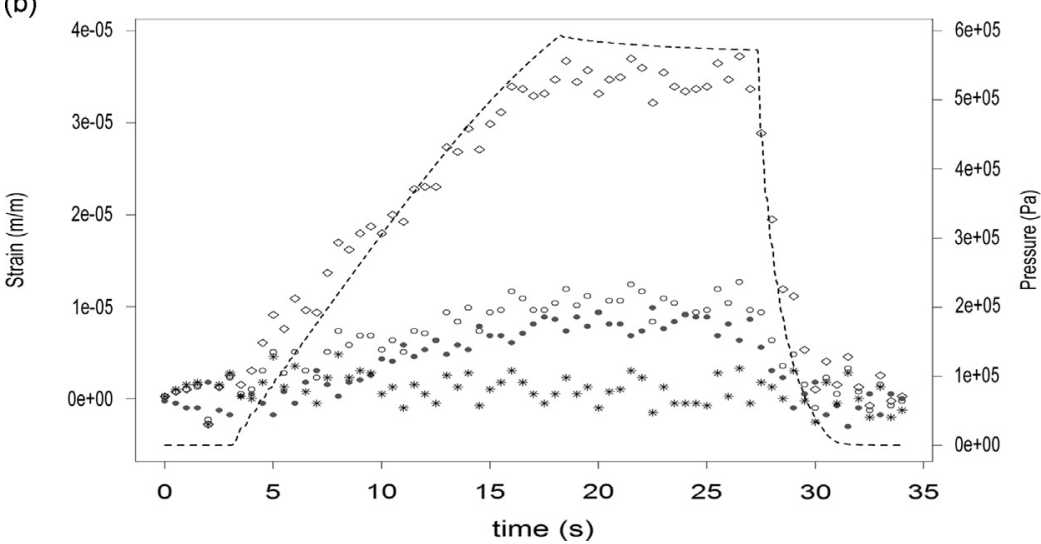

Fig. 2. Strain and pressure measurements: (a) circumferential strain test and (b) axial strain test. $\circ$ strain at top side (SG-1 and SG-4); $\diamond$ strain at outer side (SG-2 and SG-5); * strain at bottom side (SG-6); and • strain at inner side (SG-3 and SG-7). Pressure measurements in dashed lines (PT-2).

\section{Data collection}

The experimental set up is composed of a copper pipe of nominal diameter $D=0.02 \mathrm{~m}$, pipe-wall thickness $e=0.001 \mathrm{~m}$ and pipe length $L=105 \mathrm{~m}$. The torus radius is $R=0.45 \mathrm{~m}$ and 36 rings compose the entire coil, which is $1 \mathrm{~m}$ high. Seven foil strain gauges (SG), with a tolerance of $\pm 0.85 \mu \mathrm{m} / \mathrm{m}$, were installed in the middle section of the pipe in order to carry out strain measurements in the axial and in the circumferential directions for different positions of the cross-section, as depicted in Fig. 1. Three pressure transducers (PT), with pressure range of 0-25 bar and $0.2 \%$ accuracy of total range, were installed at different locations of the pipe. Young's modulus of elasticity and Poisson ratio were experimentally determined by measuring stress-strain states over a straight pipe sample for the experimental range of pressures. The obtained values were Young's modulus of elasticity $E=105 \mathrm{GPa}$ and Poisson ratio $\nu=0.33$. The hydropneumatic vessel has a capacity of 601 and the initial air volume before any test was approximately $1 / 3$ of the hydropneumatic vessel volume. Each coil ring is supported by four anchoring points over a metallic frame, where rubber supports allow the pipe movement. The measured wave speed during water-hammer tests was $1120 \mathrm{~m} / \mathrm{s}$.

When the pipe is bended to get the curved shape of the pipe coil, the cross-section is changed from circular to slightly elliptic (as shown in Sections 4 and 5). This ellipticity of the cross-section has important consequences in the structural behaviour of the system.

Two different kinds of experiments were carried out in the coil facility. Firstly, circumferential and axial strains were measured for different quasi-steady pressure loads, and secondly dynamic loading was applied by producing water-hammer events for different flow rates. The results presented in Section 5 correspond to a steady pressure test of $6 \times 10^{5}$ Pa and results in Section 6 correspond to a water-hammer wave produced for an initial flow rate of $1.4 \times 10^{-4} \mathrm{~m}^{3} / \mathrm{s}$. Fig. $2 \mathrm{shows}$ the measurements obtained for static pressure tests, namely axial and circumferential strains of the pipe-wall, and fluid pressures. 


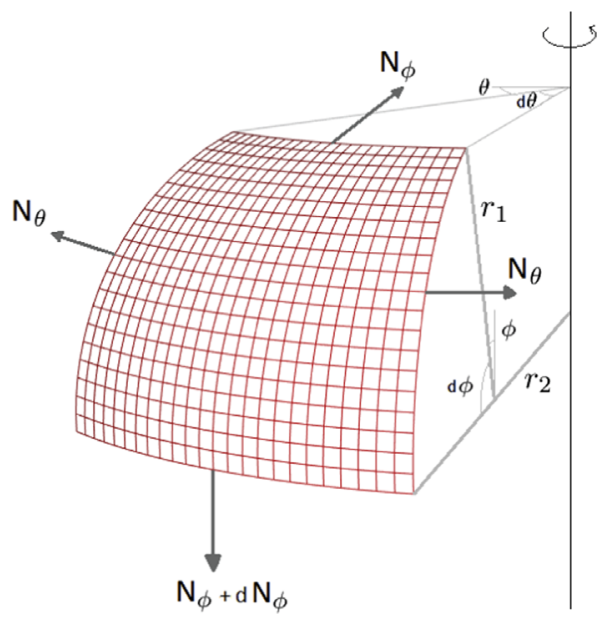

Fig. 3. Element of the axisymmetrically loaded shell of revolution.

\section{Model development}

\subsection{Membrane theory of shells of revolution: stress-strain models}

A shell of revolution is a three-dimensional structure bounded primarily by two arbitrary curved surfaces a relatively small distance apart (Seide, 1975). In the present section the membrane theory of shells of revolution, assuming thin wall and axisymmetrical load, has been considered for the computation of axial and circumferential stresses. Nonetheless, the membrane theory of shells of revolution assumes momentless shells, thus, this theory does not consider the bending of the cross-section.

The starting point to derive stress-strain models is the general solution of the membrane theory of shells of revolution for axisymmetric loads (Zingoni, 1997), which outcome is an expression for axial and circumferential stresses:

$$
\begin{aligned}
& N_{\phi}=\frac{1}{r_{2} \sin ^{2} \phi}\left[\int r_{1} r_{2}\left(p_{r} \cos \phi-p_{\phi} \sin \phi\right) \sin \phi d \phi+j\right], \\
& N_{\theta}=r_{2} p_{r}-\frac{r_{2}}{r_{1}} N_{\phi},
\end{aligned}
$$

where $N_{\phi}$ is the circumferential unit force and $N_{\theta}$ is the axial unit force; $p_{r}$ is the load applied in the normal direction of the shell midsurface, $p_{\phi}$ is the load in the tangential direction of the shell midsurface; $r_{1}$ and $r_{2}$ correspond to different radii of curvature (see Fig. 3); $\phi$ and $\theta$ are respectively the angles corresponding to the parallel and meridional directions; and $j$ is a constant of integration to be obtained from appropriate boundary conditions.

Adapting the general solution with regard to the coil geometry, two stress-strain models, one for the torus with elliptic cross-section (elliptic torus model) and the other for the circular cross-section (circular torus model), were implemented following the development explained in Zingoni (1997).

\subsubsection{Torus with elliptic cross-section}

After determining the radius of curvature $r_{1}$ and $r_{2}$ for an elliptic cross-section, and introducing them in Eq. (2), the definite integral can be solved fixing a finite $j$ constant for $\phi=0$ and $\phi=\pi$, for which $\sin \phi=0$. Expressing the unitary force in terms of stress, the expression obtained for circumferential stress is

$$
\sigma_{c}=\frac{p a^{2}}{e \sqrt{a^{2} \sin ^{2} \phi+b^{2} \cos ^{2} \phi}}\left[\frac{R \sqrt{a^{2} \sin ^{2} \phi+b^{2} \cos ^{2} \phi}+\frac{a^{2}}{2} \sin \phi}{R \sqrt{a^{2} \sin ^{2} \phi+b^{2} \cos ^{2} \phi}+a^{2} \sin \phi}\right]
$$

where $\sigma_{c}$ is the circumferential stress, $p$ is the inner pressure, $e$ is the pipe-wall thickness, $a$ is the minor semi-axis length of the elliptic cross-section, $b$ is the major semi-axis length and $R$ is the torus radius from the centre of the toroid to the pipe axis.

Substituting the previous Eq. (4) into Eq. (3) and rearranging terms the expression for axial stress is obtained:

$$
\sigma_{a}=\frac{p a^{2}}{e b^{2}}\left[R\left(\frac{b^{2}-a^{2}}{a^{2}}\right) \sin \phi+\frac{b^{2}-\frac{1}{2}\left(a^{2} \sin ^{2} \phi+b^{2} \cos ^{2} \phi\right)}{\sqrt{a^{2} \sin ^{2} \phi+b^{2} \cos ^{2} \phi}}\right]
$$

For $R \gg a \& b$, the term $\left[\left(\left(b^{2}-a^{2}\right) / a^{2}\right) \sin \phi\right]$ in Eq. (5) becomes very sensitive to ellipse eccentricity. This high sensitivity and 
the uncertainty associated to the accuracy of the measurement of the ellipticity are the main reasons why eccentricity value is calibrated in an a posteriori analysis from axial strain measurements.

\subsubsection{Torus with circular cross-section}

Circular torus equations are straightforwardly derived by simplifying Eqs. (4) and (5) for $a=b=r$, resulting the following expression for circumferential over the outer surface:

$$
\sigma_{c}=\frac{p r}{e}\left(\frac{R+\frac{r}{2} \sin \phi}{R+r \sin \phi}\right)
$$

and over the inner surface the circumferential stress is

$$
\begin{aligned}
\sigma_{c} & =\frac{p r}{e}\left(\frac{R-\frac{r}{2} \sin \phi}{R-r \sin \phi}\right), \\
\sigma_{a} & =\frac{p r}{2 e} .
\end{aligned}
$$

For $R \gg r$, Eqs. (6) and (7) can be further simplified, cancelling the second term and reaching the expression for straight pipes (Eq. (1)) used in classic water-hammer theory. For instance, in the case of the copper coil facility analysed where $R=0.5 \mathrm{~m}$ and $r=0.02 \mathrm{~m}$ the circumferential stress at $\phi=\pi / 2$ will be $\sigma_{c}=p r / e \cdot(0.981)$. Hence, applying straight pipe equation for circumferential stress only, a $2 \%$ error will be produced as much in the most unfavourable location within the cross-section. Eq. (8) shows that axial stress is independent of the coil radius $R$ and of the position angle $\phi$. Hence, contrary to an elliptic torus, axial stress is constant along the pipe wall for the circular cross-section, like a straight pipe with closed ends.

Once stresses are computed by the previous equations, either using elliptic torus or circular torus assumptions, strains can be obtained by Hooke's law for isotropic elastic materials:

$$
\begin{aligned}
\epsilon_{c} & =\frac{1}{E}\left(\sigma_{c}-\nu \sigma_{a}\right), \\
\epsilon_{a} & =\frac{1}{E}\left(\sigma_{a}-\nu \sigma_{c}\right),
\end{aligned}
$$

where $\epsilon_{c}$ and $\epsilon_{a}$ are the circumferential and axial strains, respectively, and $\nu$ is the Poisson ratio.

\subsection{Cross-sectional bending analysis: inverse method}

Membrane theory of shells of revolution assumes thin-walled shells, that is no bending moments are transmitted along the shell. However, due to the elliptic geometry of the pipe cross-section, when the fluid pressure changes, radial loads are not balanced in the coil (they are not axisymmetric any more) as its projection on the minor axis of the ellipse will not be equal to the projection on the major axis (see Fig. 4). This unbalance of forces generates bending moments that for positive pressures will tend to reduce the eccentricity of the ellipse and vice versa for negative pressures. Hence, in the case of positive pressures, the outer fibres of the upper and lower generatrices of the cross-section will be compressed and the outer fibres of the lateral generatrices stretched. For negative pressures, the effect will be the opposite.

Strain-gauges measurements give information from these outer fibres, so the real measurements will be actually a combination of circumferential strain (obtained by membrane theory of shells of revolution) plus the extra deformation at

(a)

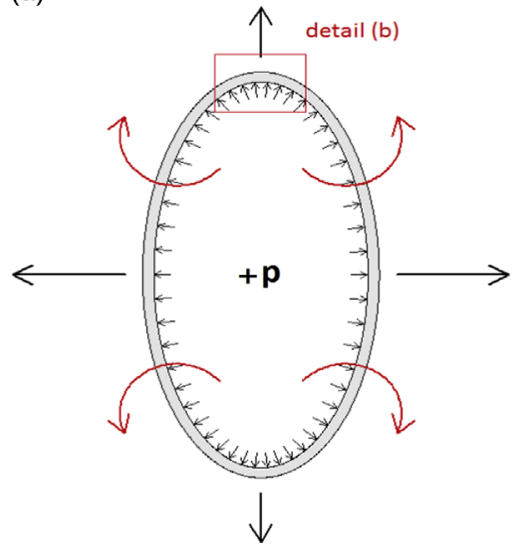

(b)

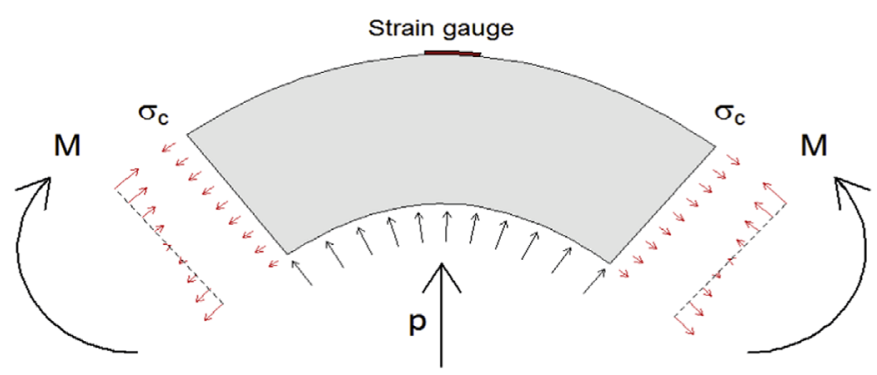

Fig. 4. Schematics of radial loads balance in (a) an elliptic cross-section and (b) detail of stresses over the upper generatrice. 


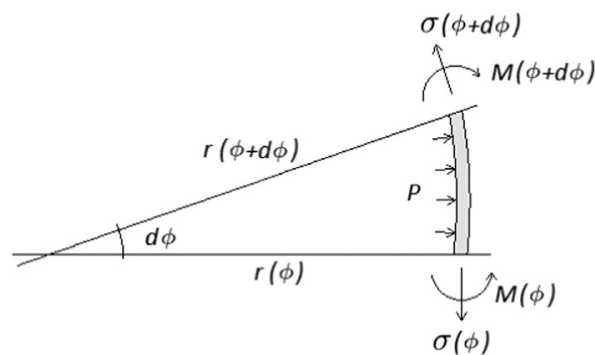

Fig. 5. Schematic for bending moment $M(\phi)$ derivation.

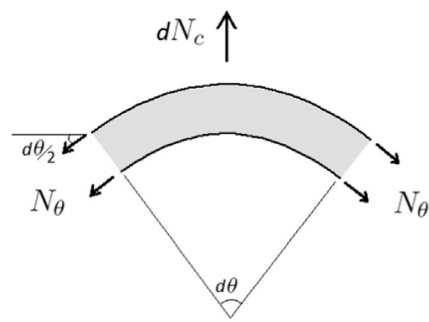

Fig. 6. Balance of forces of a control volume, pipe-wall stress forces versus centrifugal force.

the outer fibres due to bending. Therefore, the effect of bending can be analysed by an inverse method from computed circumferential strains in the central fibres of the pipe-wall and measured strains in the circumferential direction at the external fibres.

The assessment of this bending effect has two goals: firstly, it will allow the comparison between measured and computed circumferential strains, and secondly, it will provide information about the cross-sectional shape change, which is important for fluid-structure coupling during hydraulic transients in coils.

\subsubsection{Derivation of the bending moment $M$}

The pressurised elliptic cross-section is a hyperstatic system, i.e. internal forces such as the bending moment $M(\phi)$ cannot be obtained explicitly from the static equilibrium equations. Therefore, $M(\phi)$ can only be obtained numerically. The derivation carried out is based on Fig. 5.

Applying equilibrium of moments at point $(\phi+d \phi)$ and assuming some simplifications the following expression can be obtained:

$$
M(\phi+d \phi)=M(\phi)-\sigma(\phi)[r(\phi)-r(\phi+d \phi)]+P \frac{(d \phi r(\phi))^{2}}{2} .
$$

By rearranging terms in the previous equation, it yields

$$
\frac{M(\phi+d \phi)-M(\phi)}{d \phi}=-\sigma(\phi) \frac{r(\phi)-r(\phi+d \phi)}{d \phi}+P \frac{(d \phi r(\phi))^{2}}{2 d \phi},
$$

that is,

$$
\frac{d M(\phi)}{d \phi}=\sigma(\phi) \frac{d r(\phi)}{d \phi}+P \frac{d \phi r(\phi)^{2}}{2} .
$$

As the elliptic cross-section is a closed system, Eq. (13) has to be solved by imposing some internal conditions. From measurements and applying the inverse approach explained in the previous section, bending moments can be obtained at convenient points of the cross-section and, then, be used as internal conditions. Though, as the inverse method must be applied in any case, the implementation of the stress-strain models was carried out by using the inverse approach all over the cross-section.

\subsection{Centrifugal force throughout the coiled pipe}

Another effect that may contradict the axi-symmetrical loading assumption is the inertial force generated by the rotation of the flow along the coil. The centrifugal force produced by an infinitesimal control-volume of the fluid on the coil may be defined as

$$
d N_{c}=\rho d \forall \frac{V^{2}}{R}=\rho A_{f} \frac{d x}{R} V^{2}=\rho A_{f} d \theta V^{2},
$$


where $N_{c}$ is the centrifugal force, $\rho$ is the fluid density, $d \forall$ is the volume of the control-volume, $V$ is the average flow velocity and $A_{f}$ is the fluid cross-sectional area.

Applying the balance of forces shown in Fig. 6 between the axial stress of the pipe-wall and the centrifugal force $N_{c}$ :

$$
d N_{c}=2 N_{\theta} \sin \frac{d \theta}{2} \approx N_{\theta} d \theta
$$

By combining Eqs. (14) and (15), the following expression can be obtained for the axial unit force due to centrifugal effect:

$$
N_{\theta}=\rho A_{f} V^{2}
$$

which, in terms of stress, is

$$
\sigma_{a_{c}}=\frac{N_{\theta}}{A_{s}}=\rho \frac{A_{f}}{A_{s}} V^{2}=\rho \frac{\pi r^{2}}{e 2 \pi r} V^{2}=\rho \frac{r}{2 e} V^{2}
$$

where $A_{s}$ is the solid cross-sectional area.
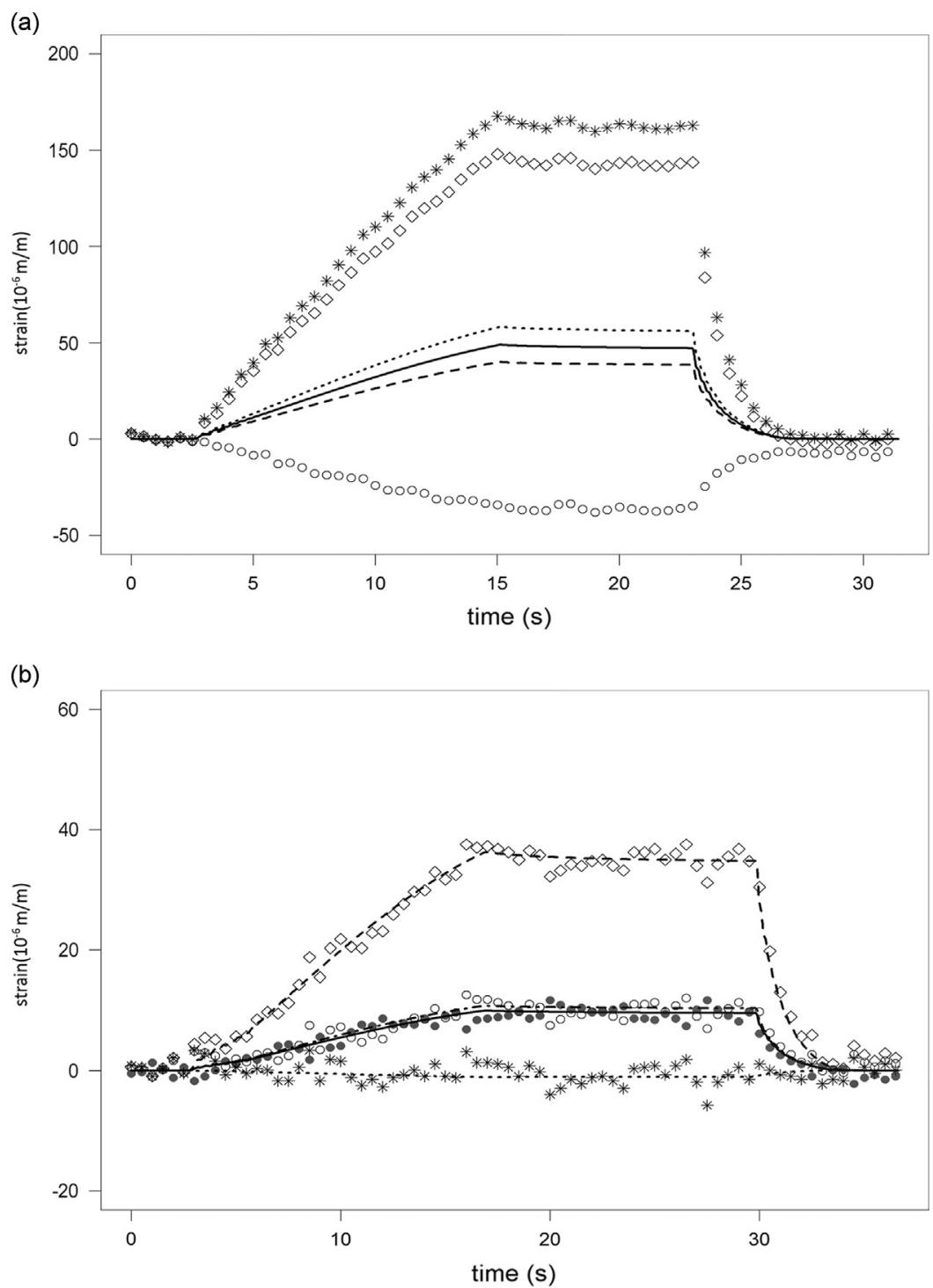

Fig. 7. Measured versus computed circumferential and axial strains using elliptic torus. $\circ$ measured strain at top side; $\diamond$ measured strain at outer side; $*$ measured strain at bottom side; • measured strain at inner side. Computed strain at top side in solid line (SG-1 and SG-4); computed strain at outer side in dashed line (SG-2 and SG-5); computed strain at bottom side in dash-dot line (SG-6); and computed strain at inner side in dotted line (SG-3 and SG-7). 
The ratio between the axial stress due to the fluid centrifugal force $\sigma_{a_{c}}$ (Eq. (17)) and the axial stress due to inner pressure $\sigma_{a_{p}}$ (Eq. (8)) is

$$
\frac{\sigma_{a_{c}}}{\sigma_{a_{p}}}=\rho \frac{V^{2}}{p} .
$$

Considering maximum effects during water-hammer events, $p_{\max }$ can be computed using Joukowsky expression $(p=\rho a V)$. Substituting in Eq. (18):

$$
\frac{\sigma_{a_{c_{\max }}}}{\sigma_{a_{p_{\max }}}}=\frac{V}{a}
$$

where $a$ is the water-hammer wave speed. In most engineering applications $a \gg V$, so the non-dimensional number presented in Eq. (19) will be very low (in the case of the copper facility $\approx 10^{-4}$ ) and, consequently, the axial stress due to inertia of the fluid flow throughout the coil can be neglected.
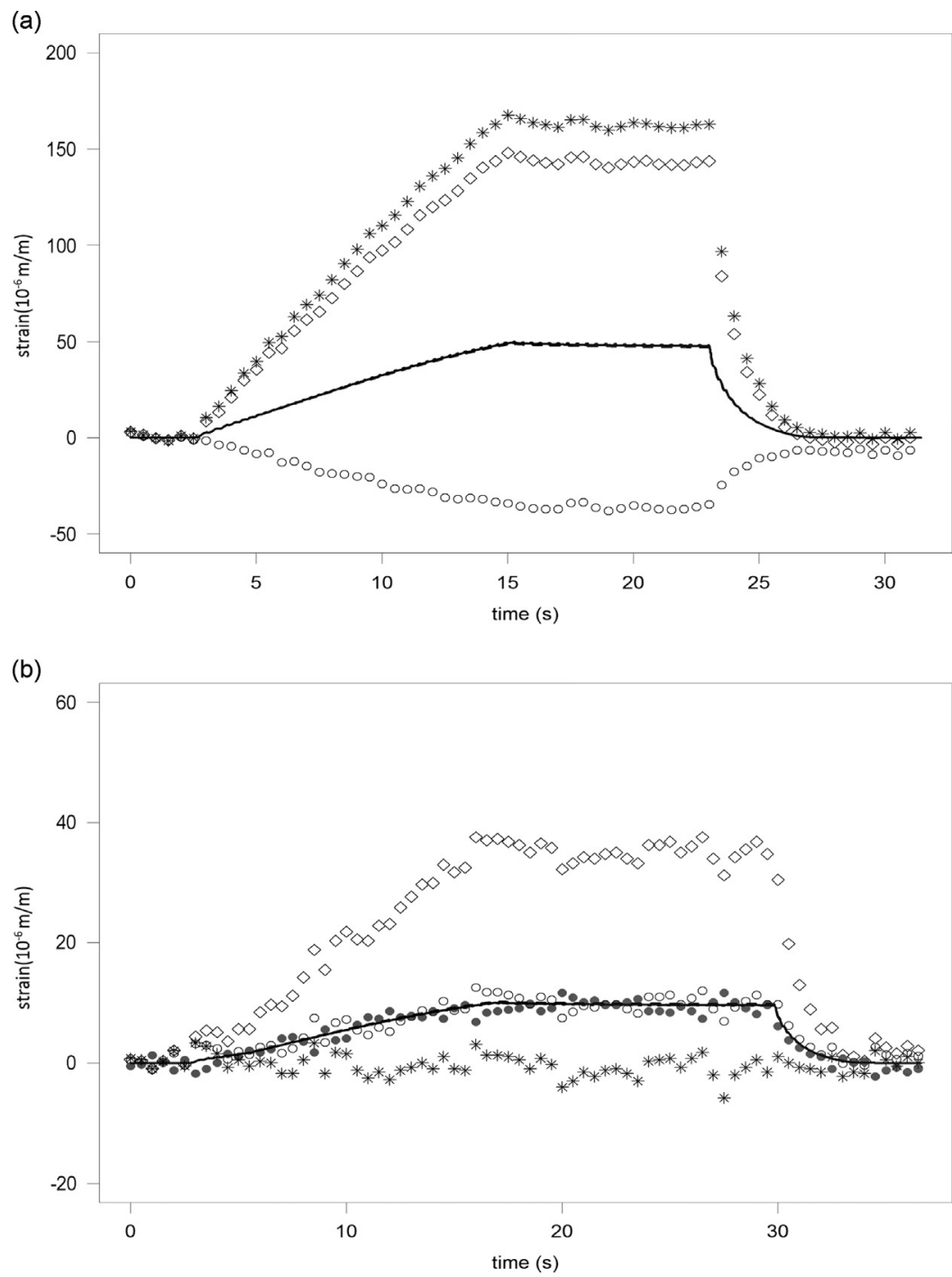

Fig. 8. Measured versus computed circumferential and axial strains for a circular torus. $\circ$ measured strain at top side; $\diamond$ measured strain at outer side; * measured strain at bottom side; • measured strain at inner side. Computed strain at top side in solid line (SG-1 and SG-4); computed strain at outer side in dashed line (SG-2 and SG-5); computed strain at bottom side in dash-dot line (SG-6); and computed strain at inner side in dotted line (SG-3 and SG-7). (a) circumferential strains and (b) axial strains. 


\section{Model application}

\subsection{Forward approach for circumferential and axial strains}

\subsubsection{Torus with elliptic cross-section}

States of stresses and strains were computed by Eqs. (4), (5), (9) and (10) for the static loading test for a pressure of $6 \times 10^{5} \mathrm{~Pa}$ (i.e., $60 \mathrm{mH}_{2} \mathrm{O}$ ) and obtained results are shown in Fig. 7 .

After adjusting ellipse eccentricity $\sqrt{\left(b^{2}-a^{2}\right) / b^{2}}=0.094$, a good agreement between the computed axial strains and the measurements is obtained. The consistency between the different positions in the cross-section strengthens the reliability of membrane theory of shells of revolution in regard to axial strains. Nevertheless, circumferential strain results do not present the same accuracy, as major discrepancies arise between circumferential strains in the different positions of the crosssection. The main reason of such discrepancies is the bending effect over the cross-sectional plane due to non-axisymmetry of loads (Fig. 4), which at this stage has not been taken into account.

\subsubsection{Torus with circular cross-section}

Stress-strain states were also solved by applying the model of the torus with circular cross-section and for the same pressure loads as in the previous section. Deformations were computed by using Eqs. (6)-(10) and the results are presented in Fig. 8.

In Fig. 8 it can be seen that both circumferential and axial computed strains from a circular torus hardly vary with their relative position in the cross-section (see all dashed lines overlapped). This is in agreement with the assumption that $R \gg r$, indicating that circumferential strains computed by classic theory do not vary much in comparison to the model for a circular torus. However, as in the case of elliptic torus, discrepancies with measurements are evident. On the other side, axial strains are constant over the crosssection, and their magnitude is close to the measured axial strain in the top and in the bottom sides of the cross-section.

In general, the circular torus model can describe quite well average circumferential and axial strains. However, the capacity of elliptic torus to adjust a posteriori elliptic eccentricity of the pipe cross-section and its reliability shown in axial strains (Fig. 7) justify a good basis to carry out the bending analysis of the cross-section. The analysis will allow the

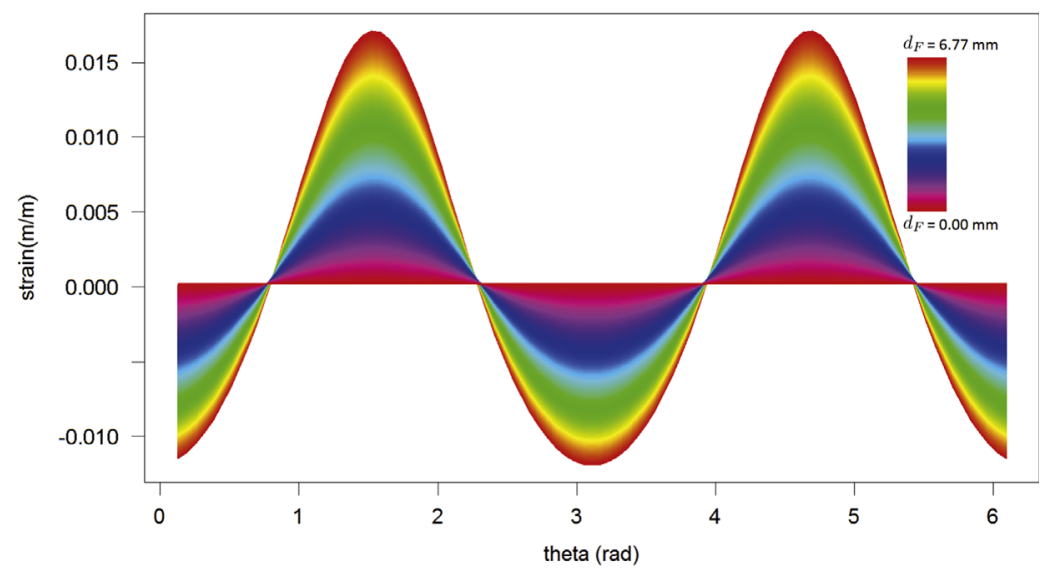

Fig. 9. Circumferential strains over the cross-section when focal distance $d_{F}$ is changed.

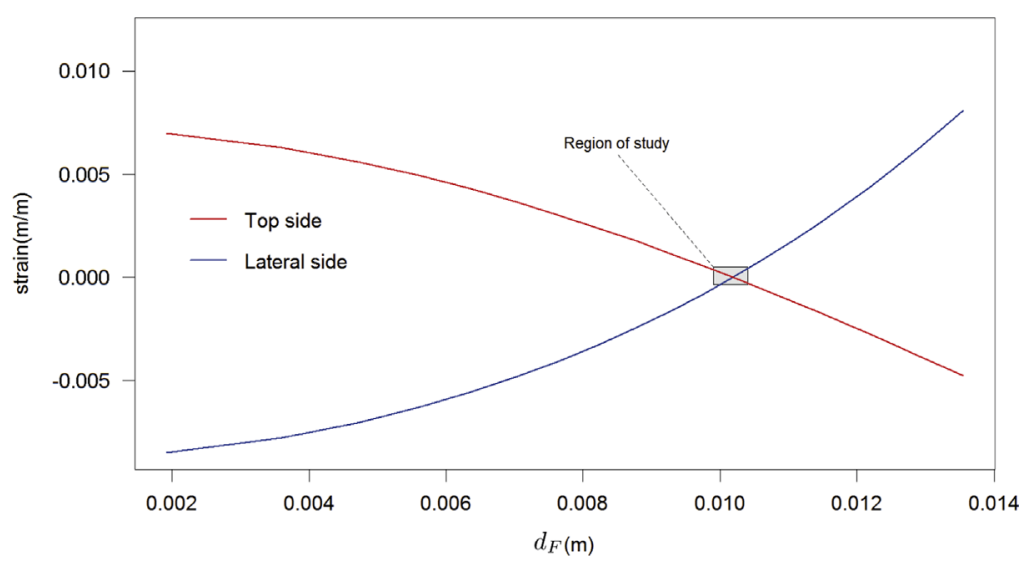

Fig. 10. Circumferential strains as a function of focal distance $d_{F}$. 


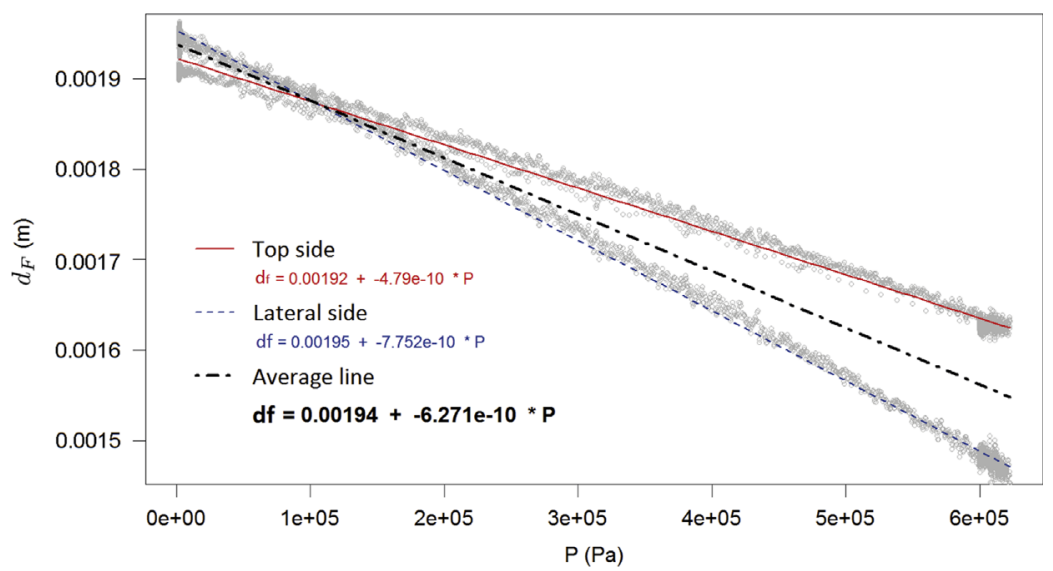

Fig. 11. Empirical laws relating pressure $p$ and focal distance $d_{F}$. (For interpretation of the references to color in this figure caption, the reader is referred to the web version of this article.)

correction of circumferential strains at the outer fibres of the pipe-wall.

It should be highlighted that the differences between measurements and computed circumferential strains by the membrane theory of shells of revolution, either considering elliptic or circular cross-section, are coherent with the phenomena explained in Section 4.2. The bending of the cross-section when pressure is increased produces a compression of the external fibres of the top side of the cross-section and stretches the external fibres in the lateral sides. Hence, computed results overestimate circumferential strains in the top side and underestimate circumferential strains in the lateral sides in comparison to measurements.

\subsection{Inverse approach for bending effects}

The inverse approach is used to correct the bending effect not described by the previous models. First of all, the relation between strains and the focal distance (from this point on $d_{F}$, i.e. distance between the two foci of the ellipse) is determined. After a geometrical development, the deformation at any point of the external side of the pipe wall has to be analytically found for any change in ellipse eccentricity. Fig. 9 shows the deformation from circumferential cross-section (i.e. $d_{F}=0$ ) to any $d_{F}$ up to a maximum value of $d_{F}=6.77 \mathrm{~mm}$. The relation between $d_{F}$ and strains at the external sides of the pipe wall for the upper and lateral generatrices of the cross-section is presented in Fig. 10. Although the general law is not linear, as the experimental strains are located in a very small range, the expression can be approximated to a linear relationship.

In a second stage, as time-series relating pressures and strains could be obtained from measurements, with the combination of the previous relations depicted in Fig. 10, an empirical relation between pressure and $d_{F}$ could be found (Fig. 11).

In Fig. 11 three empirical relationships between pressure $p$ and the focal distance $d_{F}$ are shown: the solid red line represents this relation obtained according to strains measured on the top side of the cross-section, the blue dash line according to strains measured on the lateral side and the black dash-dot line represents the average line obtained between the previous relationships. The relationship between $d_{F}$ and $p$ should be equal over the whole cross-section, consequently, the solid and dashed lines, ideally, should be overlapping. This graph allows the validation of the method: the closer the solid and dash lines are, the more accurate the method is. Notice that before this stage no calibration had been carried out, only ellipse eccentricity had been initially adjusted in order to get the best fitting for axial strains. This small discrepancy over the solid and dash lines is due to uncertainty arisen from strain measurements, experimental Young's modulus, ellipse eccentricity and other possible secondary effects, like torsion and shear along the coil due to its structural constraints. A better fitting of both lines could be achieved calibrating the mentioned parameters. Notwithstanding, obtained linear laws are quite similar, therefore for the purpose of the analysis both relationships are considered accurate enough and the dash-dot interpolated line will be used in next steps as the relationship between pressure and focal distance (Eq. (20)). No further calibration is applied.

$$
d_{F}[\mathrm{~m}]=0.00194-6.27 \cdot 10^{-10} * p[\mathrm{~Pa}] .
$$

Eq. (20) allows, therefore, the estimation of 'an average' focal distance of elliptic cross-section for all pressures within the experimental pressures range. Consequently, the volume change in the coil due to cross-section shape change can be analysed, and circumferential strains computed from membrane theory of shells of revolution can be corrected. Fig. 12 presents the strains computed and corrected with this approach (elliptic torus with bending effect), the previous strains obtained with the basic models (elliptic and circular torus) and the experimental data.

Fig. 12 shows that once circumferential strains are corrected by taking into account the bending effect, there is good agreement between measured and computed strains, particularly in the inner and outer sides of the cross-section. Discrepancies are observed in the top position of the cross-section where computed strains seem to be more sensitive to the 

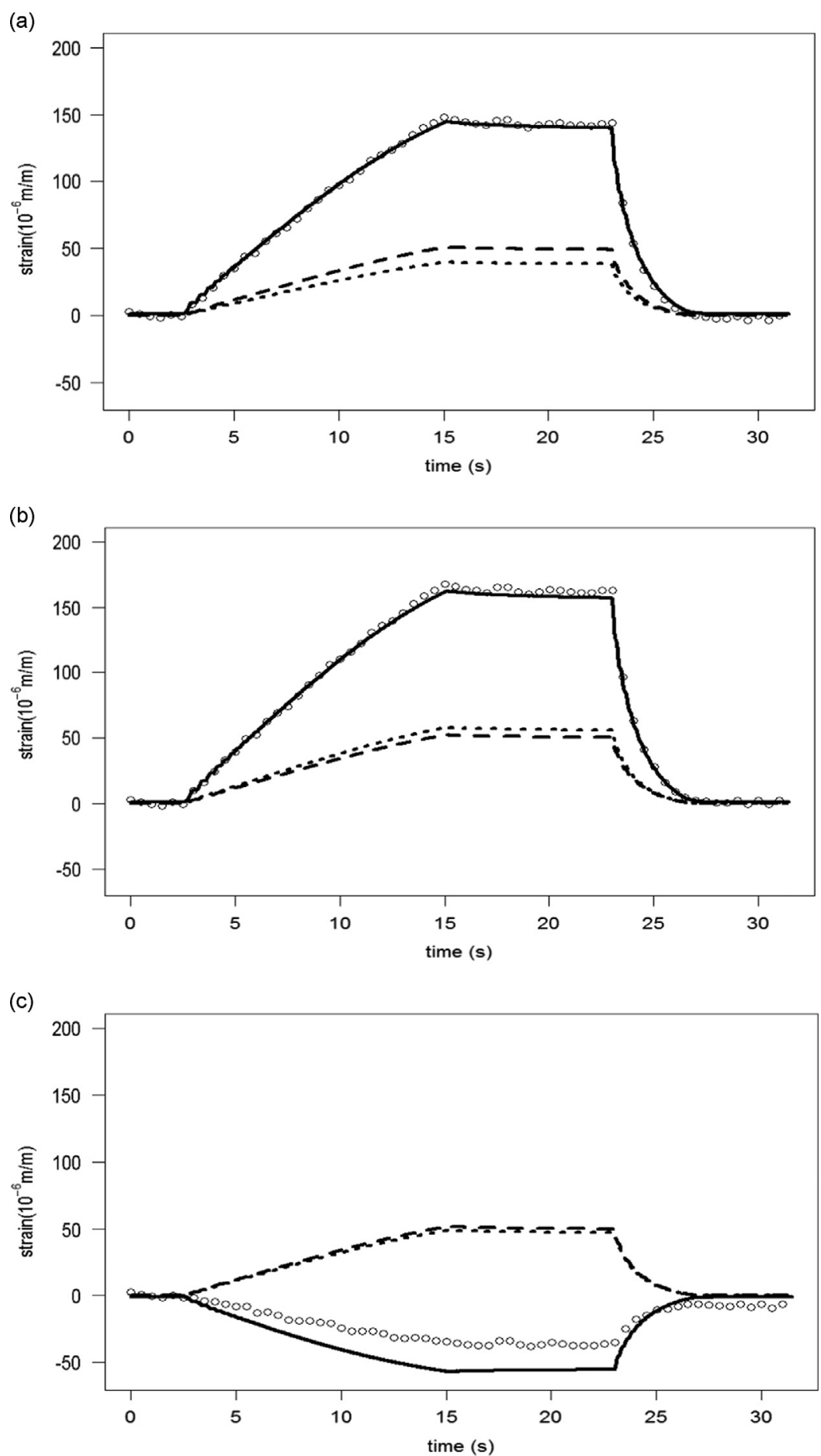

Fig. 12. Circumferential strains corrected and not corrected versus experimental data: (a) in the outer side, (b) in the inner side, and (c) in the top side of the pipe. $\circ$ measured data; output from elliptic torus model in doted lines; from circular torus model in dashed lines; and from elliptic torus model taking into account bending effect in solid lines.

inaccuracy of the method already depicted in Fig. 11. However, the overall performance of the model after the bending correction is quite satisfactory, allowing the assessment of pipe-wall displacements for the analysed range of static loadings. 


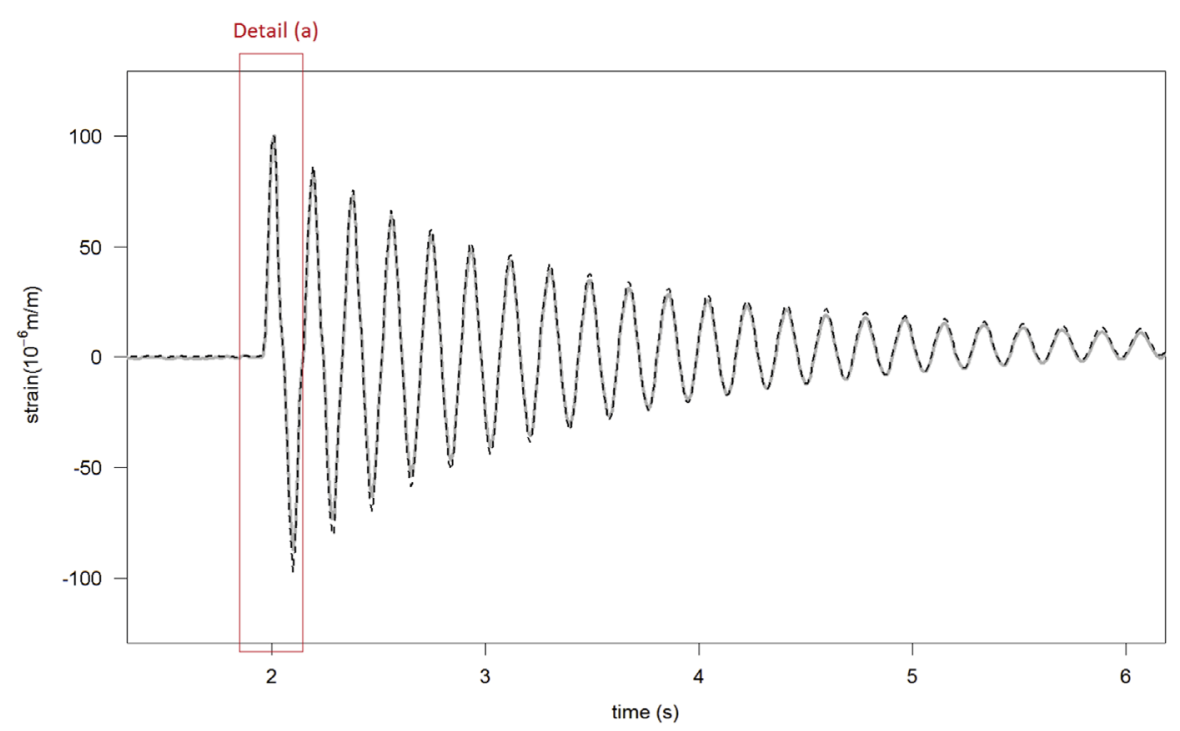

(a)

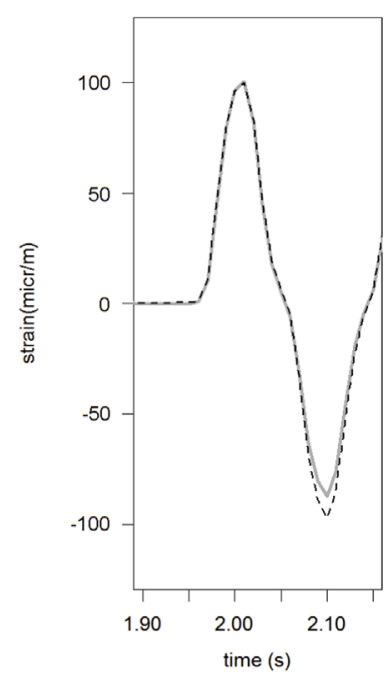

(b)
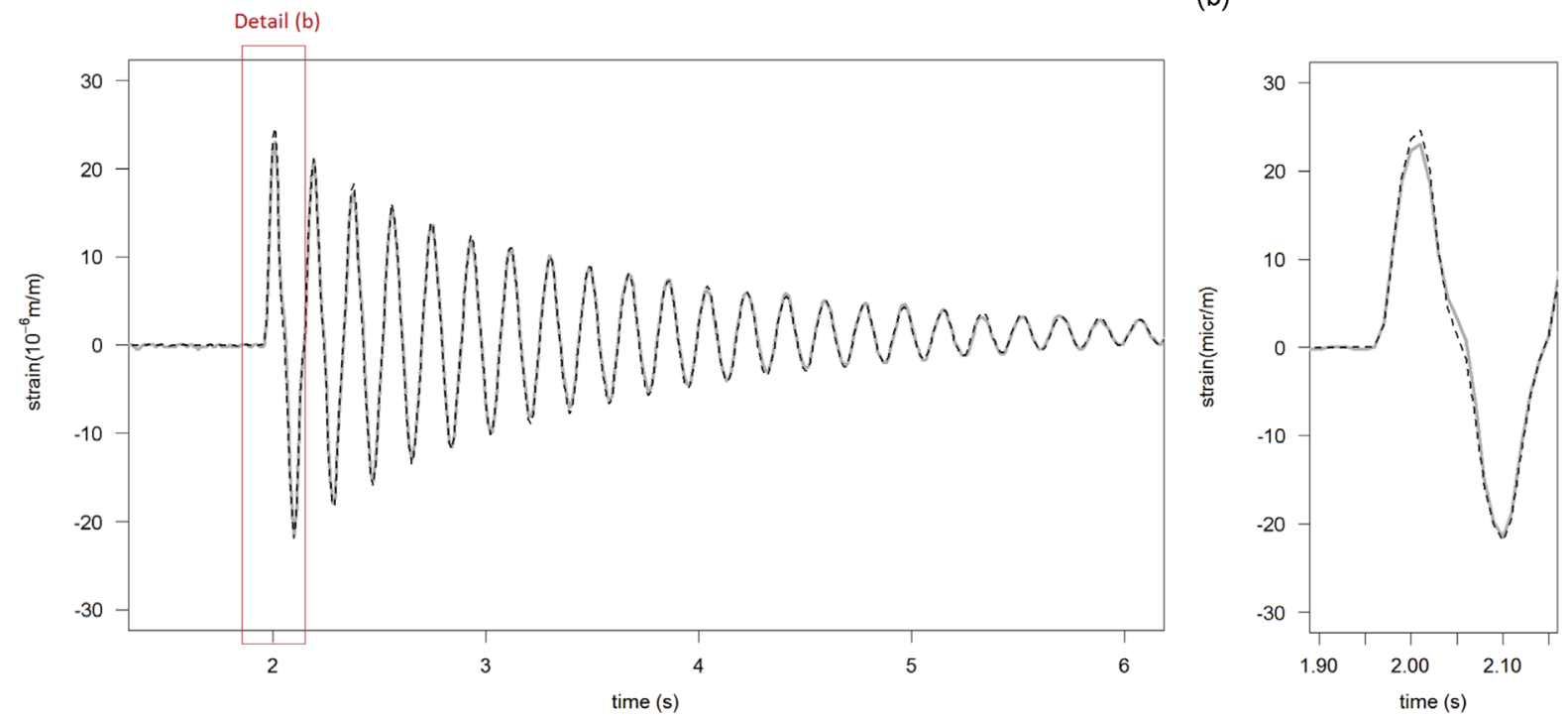

Fig. 13. Circumferential (top) and axial (down) strains (in $\mu \mathrm{m} / \mathrm{m}$ ) in the outer side of the cross-section during transient test at the middle section of the pipe and detail of the first peaks (right). Measured data in grey solid lines and computed data in black dashed lines.

\section{Model validation for dynamic loading}

Dynamic loading tests were carried out in order to assess whether the calibrated stress-strain model can accurately describe pipe-wall displacements during hydraulic transients. A hydraulic transient is characterised by fast loadingunloading cycles over the pipe wall. Consequently, other physical phenomena such as non-elastic behaviour of the copper material may arise, which are not taken into account in a static analysis.

For this purpose, once the stress-strain model was defined, strains were computed from measured pressure during a hydraulic transient test and compared with measurements. Axial strains were determined by using the elliptic model developed by membrane theory of shells of revolution approach (Eq. (5)). Circumferential strains were computed by the superposition of membrane theory (Eq. (4)) and the correction for the bending effect presented in Section 5.2.

A hydraulic transient was generated by the fast closure of a valve (in $0.1 \mathrm{~s}$ ) located at the downstream end of the pipe and for an initial discharge of $1.4 \times 10^{-4} \mathrm{~m}^{3} / \mathrm{s}$. With a frequency of data sampling of $100 \mathrm{~Hz}$ the measured wave speed was $1120 \mathrm{~m} / \mathrm{s}$. Fig. 13 presents the numerical results versus measurements of circumferential and axial strains on the outer side of the coil cross-section.

Strains computed in the circumferential direction have a Mean Squared Error equal to $2.1 \mu \mathrm{m} / \mathrm{m}$, while strains in axial direction have $0.25 \mu \mathrm{m} / \mathrm{m}$. The stress-strain model has a better performance in the axial direction because the computed 
strains in this direction have not to be corrected, since assumptions from membrane theory of shells of revolution are consistent with the physical phenomena. This is because bending is only occurring over the cross-sectional plane, and not over the horizontal plane. However, strains in the circumferential direction are affected by the bending of the elliptic crosssection (Fig. 4), and therefore uncertainty arises from the introduced correction.

\section{Simplification of pipe stress-strain equations for FSI coupling}

In FSI implementation for the analysed coil pipe system, membrane theory of shells of revolution have to be applied for circumferential and axial strains computation, while the bending effect has to be considered by the empirical relationship from Fig. 11. Coupling will be performed for 1D hydraulic transient equations. Hence, for the sake of FSI implementation, the stress-strain equations are simplified with the aim to consider only averaged values over the entire cross-section.

\subsection{Simplification for circumferential stress}

The circumferential stress equation for elliptic torus can be simplified by assuming quasi-circular cross-section $(a \simeq b \simeq r)$ and quasi-straight pipe $(R \gg r)$. Hence, substituting $a$ and $b$ by $r$ in Eq. (4):

$$
\sigma_{c_{1}}=\frac{p r^{2}}{e \underbrace{\sqrt{r^{2} \sin ^{2} \phi+r^{2} \cos ^{2} \phi}}_{r}}\left[\frac{R \sqrt{r^{2} \sin ^{2} \phi+r^{2} \cos ^{2} \phi}+\frac{r^{2}}{2} \sin \phi}{R \sqrt{r^{2} \sin ^{2} \phi+r^{2} \cos ^{2} \phi}+r^{2} \sin \phi}\right]=\frac{p r}{e} \frac{R r+\frac{r^{2}}{2} \sin \phi}{\underbrace{\frac{R+r^{2} \sin \phi}{r}}_{1 \text { for } R \gg r} \sigma_{c_{1}}}=\frac{p r}{e}
$$

obtaining the same expression corresponding to circumferential stress in straight pipes used in classic water-hammer theory.

\subsection{Simplification for axial stress}

Though, regarding axial stress the term $R\left(\left(b^{2}-a^{2}\right) / a^{2}\right) \sin \phi$ from Eq. (5) cannot be simplified. Using the same assumptions, as for the quasi-straight pipe assumption $(R \gg r)$ the term tends to $\pm \infty$ while for the quasi-circular section assumption $(a \simeq b)$ it will tend to 0 , thus the term is undefined. Hence, substituting in the other terms $a$ and $b$ by $r$ it yields

$$
\sigma_{a}=\frac{p}{e r}[R \overbrace{\left(\frac{b^{2}-a^{2}}{a^{2}}\right)}^{\text {eccentricity }} \sin \phi+\frac{r^{2}-\frac{1}{2} \overbrace{\left(r^{2} \sin ^{2} \phi+r^{2} \cos ^{2} \phi\right)}^{r^{2}}}{\sqrt{r^{2} \sin ^{2} \phi+r^{2} \cos ^{2} \phi}}] \sigma_{a}=\frac{p r}{2 e}+\frac{p R}{e}\left(\frac{b^{2}-a^{2}}{a^{2}} \sin \phi\right) .
$$

The first term of the obtained Eq. (22) is equivalent to the axial stress for circular torus, which actually is the result of the structural constraint of closed torus (same expression would be obtained for a straight pipe with frequent expansion joints and closed ends). The second term is composed of the product of two factors. The first factor ( $p R / e$ ) is equivalent to an average circumferential stress computed over the horizontal plane instead of the vertical $(R$ instead of $r)$. The last factor can be seen as a term dependent on the eccentricity of the elliptic cross-section and the position angle $\phi$. Hence, axial stress, with an averaged value of $\sigma_{a}=p r / 2 e$ varies, depending on the position over the cross-section, according to the product of the equivalent average circumferential stress over the horizontal plane multiplied by the ellipse eccentricity and projected over the horizontal plane. Accordingly, for FSI, averaged axial stress can be described by

$$
\sigma_{a}=\frac{p r}{2 e}
$$

which is the same expression as for torus with circular cross-section (see Eq. (8)).

\subsection{Simplification for bending effect}

As mentioned before, axial and circumferential stresses can be simplified to average values using reasonable assumptions according to the geometry of the coil system. To get average strain values from the bending effect, a different approach has to be chosen. The aim is to represent the increase of cross-section area when ellipticity is changed due to pressure changes. For this purpose, an equivalent radial expansion of the cross-section can be assumed and related with the $d_{F}-P$ expression determined in Section 5.2.

First of all, $d_{F}$ must be related to the ellipse area for a fixed ellipse perimeter, second, the ellipse area can be related to an equivalent radius for a circular section and, finally, the empirical $d_{F}-P$ law can be embedded.

The ellipse properties are defined as follows:

$$
\begin{aligned}
& d_{F}=2 \sqrt{b^{2}-a^{2}}, \\
& A_{\text {ellipse }}=\pi a b,
\end{aligned}
$$




$$
P_{\text {ellipse }}=2 \pi \sqrt{\frac{b^{2}+a^{2}}{2}} .
$$

For known values of $d_{F}$ and of the initial perimeter $\left(P_{0}\right)$, the previous system of equations can be simplified obtaining the following expression:

$$
A_{\text {ellipse }}=\pi \sqrt{\left(\frac{P_{0}}{2 \pi}\right)^{4}-\frac{1}{4}\left(\frac{d_{F}}{2}\right)^{4}} .
$$

Assuming the equivalent area for a circular section, the previous expression can be expressed in terms of radius:

$$
r=\sqrt[4]{\left(\frac{P_{0}}{2 \pi}\right)^{4}-\frac{1}{4}\left(\frac{d_{F}}{2}\right)^{4}} .
$$

Eq. (26) can be further simplified applying a linearisation via Taylor series ${ }^{1}$ with a centre point at $d_{F}$ corresponding, in the case of the dynamic test, to the initial steady state before closing the valve:

$$
r=0.01-1.0567 \times 10^{-4} \cdot d_{F} .
$$

In terms of circumferential strain:

$$
\epsilon_{c_{2}}=\frac{0.01-1.0567 \times 10^{-4} \cdot d_{F}}{r_{0}}-1 .
$$

Finally, substituting the empirical expression found for $d_{F}$ (Eq. (20)) in Eq. (28), it yields

$$
\epsilon_{c_{2}}=-6.169 \times 10^{-7}+6.626 \times 10^{-12} \cdot p .
$$

\subsection{Simplified strain equations}

Applying Hooke's law for isotropic materials (Eqs. (9) and (10)) and the Boltzman superposition principle for circumferential strains, the final simplified equations, in terms of strain, are

$$
\begin{aligned}
\epsilon_{a} & =\frac{1}{E}\left(\frac{p r}{2 e}-\nu \frac{p r}{e}\right), \\
\epsilon_{c} & =\frac{1}{E}\left(\frac{p r}{e}-\nu \frac{p r}{2 e}\right)-6.169 \times 10^{-7}+6.626 \times 10^{-12} p .
\end{aligned}
$$

\subsection{Fluid wave celerity in a coiled pipe}

The fluid wave celerity inside of a coil with no anchorages can be obtained by deriving the basic conservation waterhammer equations, starting by Reynolds Transport Theorem and applying the specific stress-strain equations for a coiled pipe.

Developing the mass conservation equation as explained in Chaudhry (1987) and with the update of the new circumferential strain equation (Eq. (31)), the expression reached is

$$
\frac{\partial V}{\partial x}+\left(\frac{1}{K}+(2-\nu) \frac{r}{E e}+1.3252 \times 10^{-11}\right) \frac{d p}{d t}=0,
$$

where $K$ is the bulk modulus of elasticity of the fluid and $x$ represents space in the axial direction and with the following definition:

$$
\frac{1}{\rho_{f} a_{f}}=\left(\frac{1}{K}+(2-\nu) \frac{r}{E e}+1.3252 \cdot 10^{-11}\right)
$$

\footnotetext{
${ }^{1}$ Taylor series:

$$
f(x)=\sum_{n=0}^{\infty} \frac{f^{(n)}(a)}{n !}(x-a)^{n},
$$
}

and for the analysed case:

$$
f\left(d_{F}\right)=f\left(d_{F_{0}}\right)+\frac{f^{\prime}\left(d_{F_{0}}\right)}{1 !}\left(d_{F}-d_{F_{0}}\right)+\frac{f^{\prime \prime}\left(d_{F_{0}}\right)}{2 !}\left(d_{F}-d_{F_{0}}\right)^{2}+\frac{f^{\prime \prime \prime}\left(d_{F_{0}}\right)}{3 !}\left(d_{F}-d_{F_{0}}\right)^{3}+\cdots
$$


the fluid wave celerity can be obtained:

$$
a_{f}=\sqrt{\left[\left(\frac{1}{K}+(2-\nu) \frac{r}{E e}+1.3252 \cdot 10^{-11}\right) \rho_{f}\right]^{-1}}
$$

It should be highlighted that, in the previous Eq. (34), the constant value associated to the bending effect $\left(1.3252 \times 10^{-11} \mathrm{~m} / \mathrm{Pa} \mathrm{s}\right)$ is specific of this case study and cannot be generalised for other pipe systems with different sizes, coil radii and pipe materials. The remaining terms can be generalised to any pipe coil.

Finally substituting values according to the coil parameters the wave celerity obtained is $1261 \mathrm{~m} / \mathrm{s}$, while omitting the cross-sectional bending term the wave celerity would be $1274.5 \mathrm{~m} / \mathrm{s}$. For instance, for a rigid conduit, the celerity is $1480 \mathrm{~m} / \mathrm{s}$, for an elastic straight thin-walled pipe anchored against longitudinal movement in its upper end, $a=1292 \mathrm{~m} / \mathrm{s}$ and, finally, allowing the movement in both boundaries, the celerity is $1260 \mathrm{~m} / \mathrm{s}$.

\section{Validation of the simplified equations}

\subsection{Pipe displacements using the elliptic torus model and the simplified equations}

In order to assess the effect of circumferential, axial and bending deformations in the context of hydraulic transients, pipe-wall displacements were first computed from the stress-strain model for the static pressure test. Fig. 14 depicts such displacements either over the cross-sectional and horizontal planes of the torus for a static pressure of $6 \times 10^{5}$ Pa. For the sake of visual representation, strains have been amplified by a scale factor of 10 in regard to bending, 100 for circumferential strains and 10000 for axial strains.

The total deformation of the pipe section and pipe length for the pressurised system has been computed in order to get an idea of the magnitude of circumferential and axial strain effects and also the cross-sectional shape change due to bending. The computation has been carried out for both, elliptic torus before and after simplification. Eqs. (35)-(37) describe the volume variation either by circumferential, axial and bending deformations:

$$
\begin{aligned}
& \Delta V_{c}=L \cdot \int_{\phi=0}^{\phi=2 \pi} \epsilon_{c_{(\phi)}} \cdot \frac{1}{k_{(\phi)}^{2}} d \phi, \\
& \Delta V_{a}=\left.L \cdot \overline{\epsilon_{a}}\right|_{c s}, \\
& \Delta V_{d_{F}}=L \cdot\left(\pi \sqrt{\left(\frac{P_{0}}{2 \pi}\right)^{4}-\frac{1}{4}\left(\frac{d_{F}}{2}\right)^{4}}-A_{0}\right),
\end{aligned}
$$

where $\Delta V_{c}$ is the volume change due to circumferential strain, $\Delta V_{a}$ due to axial strain, and $\Delta V_{d_{F}}$ due to bending effect, $k$ is the curvature of the ellipse which is dependent on the angle $\phi, L$ is the total pipe length, $P_{0}$ is the initial perimeter of the

(a)

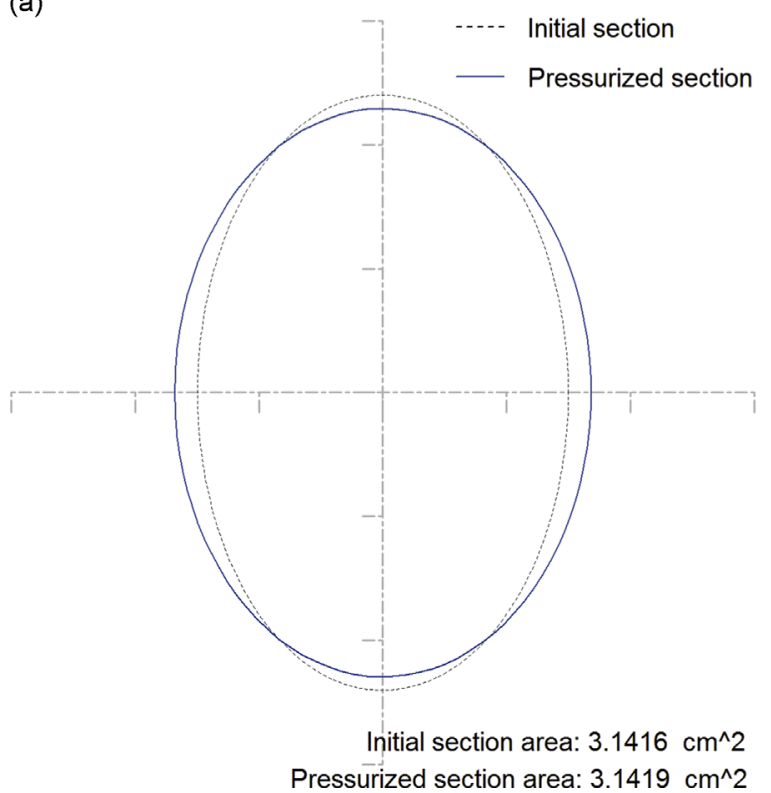

(b)

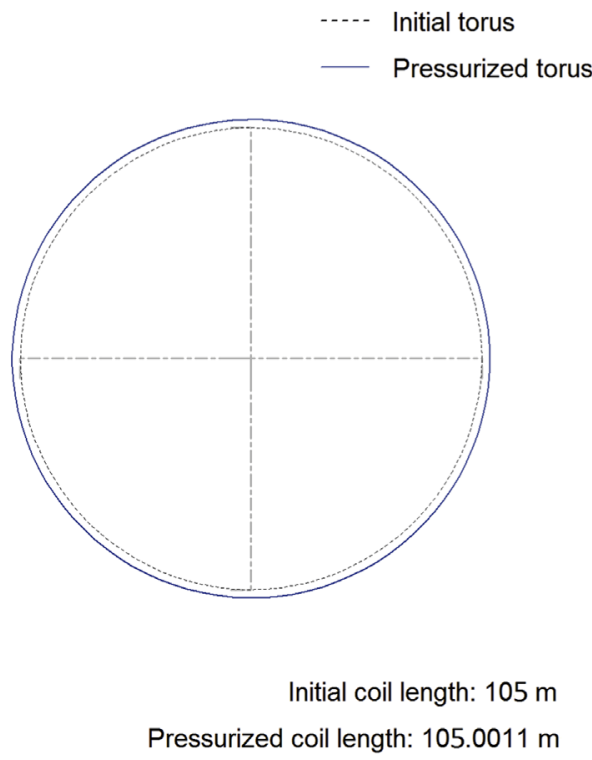

Fig. 14. Computed displacements of the pipe wall over the coil cross-section (left) and in the horizontal plane (right). 
Table 1

Volume change for a steady pressure of $6 \times 10^{5} \mathrm{~Pa}$ (i.e. $60 \mathrm{~m}$ ).

\begin{tabular}{lllr}
\hline Volume change & Non-simplified equations & Simplified equations & Error (\%) \\
\hline$\Delta V_{c}$ & $3.24 \times 10^{-6}$ & $3.11 \times 10^{-6}$ & -4 \\
$\Delta V_{a}$ & $3.60 \times 10^{-7}$ & $3.16 \times 10^{-7}$ & -12 \\
$\Delta V_{d_{F}}$ & $1.64 \times 10^{-7}$ & $2.19 \times 10^{-7}$ & 33 \\
Total $\Delta V$ & $3.76 \times 10^{-6}$ & $3.70 \times 10^{-6}$ & -3 \\
\hline
\end{tabular}

cross-section and $A_{0}$ is the initial area.

Results of the application of previous equations are shown in Table 1. In both cases, approximately 85\% of the total volume variation when the pipe is pressurised corresponds to circumferential expansion of the conduit, 8-10\% to the axial deformation and $4-6 \%$ to the bending of the cross-section. The volume variation inside the pipe is more sensitive to circumferential strain effect than to axial strain or bending effects, even though, these minor effects cannot be neglected The simplified equations give similar results in comparison to the original elliptic torus equations.

\subsection{Range of application}

The first criteria to determine the range of application of the presented equations is the consideration of the limit between thin and thick pipe-wall. In the current study, Goldberg et al. (1974) criteria are assumed, in which thin and thick pipe-wall theories were compared for circular cylinders submitted to inner pressure loads, concluding that for ratios $r / e>10$ results using thin-walled assumption were satisfactory.

In the simplification of the equations in Section 7.3, two extra assumptions are taken: quasi-circular cross-section and quasi-straight pipe. As it was shown in Fig. 8, the circular torus assumption presents good average values of axial strains, while the quasi-straight pipe assumption only affects distribution of circumferential stresses over the cross-section, but not its average value. Therefore, in the context of 1D hydraulic transient modelling, where only averaged strain values are concerned, the elliptic eccentricity of the cross-section does not represent any constraint in regard to the range of validity of the proposed equations for axial and circumferential strains.

Additionally, the bending analysis carried out is only valid for the coil system analysed. The empirical relation between $d_{F}$ and $P$ (Eq. (20)) must be redefined if structural conditions are changed. The presented equation for equivalent circumferential strain due to bending (Eq. (29)) is valid only for the tested range of pressures (approx. $0-10^{6} \mathrm{~Pa}$ ), as the linearisation via Taylor series is centred in $d_{F}$ corresponding to a non-pressurised system; consequently, the greater the amplitude of pressure range is, the greater the error will be.

\section{Summary and conclusions}

Two stress strain models based on the theory of shells of revolution are presented to describe the stress-strain relationship in the cross-section of a coiled pipe. A semi-empiric bending analysis has been carried out in order to improve the accuracy of the models.

Membrane theory of shells of revolution applied to an elliptic torus has been proven to be a good approach for the axial strain description but inaccurate with regard to circumferential strain. The main reason is that circumferential strains are strongly affected by a common singularity of coiled pipes: the cross-section is slightly elliptic and bending moments are generated over the cross-sectional plane. In order to assess the bending effect and correct circumferential strains, a semiempiric method has been proposed to determine an empiric law relating the ellipse focal distance with pipe inner pressure. The method enables the assessment of cross-sectional shape change and the correction of circumferential strains. The effect of the centrifugal force produced by the rotating flow within the coil has been also assessed, concluding that its effect is negligible in comparison to the inner pressure effect. Validation has been carried out for dynamic loading with the aim to ensure the final purpose of the research, FSI during hydraulic transients. Finally, for the sake of FSI implementation, a simplification of the proposed model is presented.

Displacement volumes have been computed for the static pressure concluding that the impact of the three effects (circumferential, axial and bending strains) are important to describe hydraulic transients in pipe-coils and they should be distinguished for FSI assessment. Circumferential and axial strains have to be described by Eqs. (4), (5), (9) and (10). Displacements due to bending (i.e. cross-sectional shape change) can be directly obtained from the empirical law relating ellipse focal distance with pressure depicted in Eq. (20). The simplified version of the equations for FSI implementation is given in Eqs. (31) and (30). The range of validity of these equations, according to the membrane theory of shells of revolution, is subjected to thin-wall criteria (i.e. $r / e>10$ ), while the presented bending analysis, due to its empirical nature, is only valid for the test facility and range of pressures assessed. 


\section{Acknowledgements}

This research is supported by the Portuguese Foundation for Science and Technology (Fundação para a Ciência e a Tecnologia) through the Project ref. PTDC/ECM/112868/2009 "Friction and mechanical energy dissipation in pressurised transient flows: conceptual and experimental analysis" and the Ph.D. grant ref. SFRH/BD/51932/2012 also issued by FCT under IST-EPFL joint Ph.D. initiative.

\section{References}

Anderson, A., Johnson, G.R., 1990. Effect of tube ovalling on pressure wave propagation speed. Proceedings of the Institution of Mechanical Engineers, Part H: Journal of Engineering in Medicine 204 (4), 245-251.

Bergant, A., Tijsseling, A.S., Vítkovsky`, J.P., Covas, D.I., Simpson, A.R., Lambert, M.F., 2008a. Parameters affecting water-hammer wave attenuation, shape and timing, Part 1: mathematical tools. Journal of Hydraulic Research 44 (3), 373-381.

Bergant, A., Tijsseling, A.S., Vítkovsky`, J.P., Covas, D.I., Simpson, A.R., Lambert, M.F., 2008b. Parameters affecting water-hammer wave attenuation, shape and timing, Part 2: case studies. Journal of Hydraulic Research 46 (3), 382-391.

Brown, F.T., Tentarelli, S.C., 2001a. Dynamic behavior of complex fluid-filled tubing systems, Part 1: tubing analysis. Journal of Dynamic Systems, Measurement, and Control 123 (1), 71-77.

Brown, F.T., Tentarelli, S.C., 2001b. Dynamic behavior of complex fluid-filled tubing systems, Part 2: system analysis. Journal of Dynamic Systems, Measurement, and Control 123 (1), 78-84.

Chaudhry, H.M., 1987. Applied Hydraulic Transients. Van Nostrand Reinhold, New York.

Clark, R., Reissner, E., 1950. Deformations and stresses in Bourdon tubes. Journal of Applied Physics 21 (12), $1340-1341$.

Goldberg, J.E., Korman, T., Baluch, M.H., 1974. On the use of various shell theories in the analysis of axisymmetrically loaded circular containers. Nuclear Engineering and Design 30 (1), 88-92.

Seide, P., 1975. Small Elastic Deformations of Thin Shells. Noordhoff International Pub.

Skalak, R., 1955. An Extension of the Theory of Water Hammer. Dept of Civil Engineering and Engineering Mechanics, Columbia University, New York.

Tijsseling, A., 1996. Fluid-structure interaction in liquid-filled pipe systems: a review. Journal of Fluids and Structures 10 (2), $109-146$.

Tijsseling, A., 2007. Water hammer with fluid-structure interaction in thick-walled pipes. Computers \& Structures 85 (11), $844-851$.

Zingoni, A., 1997. Shell Structures in Civil and Mechanical Engineering: Theory and Closed-form Analytical Solutions. Thomas Telford, London. 